\title{
Long-read and chromosome-scale assembly of the hexaploid wheat genome achieves higher resolution for research and breeding
}

Jean-Marc Aury ${ }^{1, *}$, Stefan Engelen ${ }^{1}$, Benjamin Istace ${ }^{1}$, Cécile Monat ${ }^{2}$, Pauline Lasserre-Zuber ${ }^{2}$, Caroline Belser ${ }^{1}$, Corinne Cruaud $^{3}$, Hélène Rimbert ${ }^{2}$, Philippe Leroy $^{2}$, Sandrine Arribat ${ }^{4}$, Isabelle Dufau ${ }^{4}$, Arnaud Bellec ${ }^{4}$, David Grimbichler ${ }^{5}$, Nathan Papon ${ }^{2}$, Etienne Paux ${ }^{2}$, Marion Ranoux ${ }^{2}$, Adriana Alberti ${ }^{1,7}$, Patrick Wincker ${ }^{1}$, Frédéric Choulet ${ }^{2, *}$

${ }^{*}$ corresponding authors

${ }^{1}$ Génomique Métabolique, Genoscope, Institut François Jacob, CEA, CNRS, Univ Evry, Université Paris-Saclay, 91057 Evry, France

2 GDEC, Université Clermont Auvergne, INRAE, UMR1095, 63000 Clermont-Ferrand, France

${ }^{3}$ Commissariat à l'Energie Atomique (CEA), Institut François Jacob, Genoscope, F-91057 Evry, France

${ }^{4}$ INRAE, CNRGV French Plant Genomic Resource Center, F-31320, Castanet Tolosan, France

${ }^{5}$ Mésocentre Clermont Auvergne, DOSI / Bâtiment Turing, 7 avenue Blaise Pascal, 63178 Aubière CEDEX

${ }^{6}$ Current address: Université Paris-Saclay, CEA, CNRS, Institute for Integrative Biology of the Cell (I2BC), 91198, Gif-sur-Yvette, France. 


\section{Abstract}

The sequencing of the wheat (Triticum aestivum) genome has been a methodological challenge for many years due to its large size $(15.5 \mathrm{~Gb})$, repeat content, and hexaploidy. Many initiatives aiming at obtaining a reference genome of cultivar Chinese Spring have been launched in the past years and it was achieved in 2018 as the result of a huge effort to combine short-read whole genome sequencing with many other resources. Reference-quality genome assemblies were then produced for other accessions but the rapid evolution of sequencing technologies offers opportunities to reach high-quality standards at lower cost. Here, we report on an optimized procedure based on long-reads produced on the ONT (Oxford Nanopore Technology) PromethION device to assemble the genome of the French bread wheat cultivar Renan. We provide the most contiguous and complete chromosome-scale assembly of a bread wheat genome to date, a resource that will be valuable for the crop community and will facilitate the rapid selection of agronomically important traits. We also provide the methodological standards to generate high-quality assemblies of complex genomes.

\section{Introduction}

Bread wheat (Triticum aestivum) is among the most important cereal crops and a better knowledge in the area of wheat genomics is needed to face the main challenge of ensuring food security to a growing population in the context of climate change. Improving productivity requires both that local producers adapt their practices to increase their climate resilience and a better understanding of the wheat production systems. In this context, a better knowledge of the wheat genome and its gene content, but also the sequencing of numerous accessions, are essential.

However, the genome of bread wheat is particularly characterized by its complexity. Indeed this hexaploid genome is the result of two interspecific hybridization events. The earliest cultivated wheat was diploid, but humans have intensified the cultivation of polyploid species. Recent studies show that these polyploid species appear to be advantaged by their genomic plasticity ${ }^{1}$. Indeed, modifications of the gene space and related elements are buffered by the polyploid nature of wheat and open a wider field to selection. Bread wheat is composed of three subgenomes A, B and D derived from three ancestral diploid species that diverged between 2.5 and 6 million years ago².

The wheat genome is one of the largest among sequenced plant genomes (15.5 Gb), mainly composed of repetitive sequences (ca. $>85 \%$ ), and contains many homoeologous regions 
between the three subgenomes ( $A, B$ and $D$ ). Repetitive sequences and polyploidy pose serious challenges in the generation of genome assemblies. The adventure of sequencing the hexaploid wheat genome began in 2005 with the creation of the International Wheat Genome Sequencing Consortium (IWGSC) ${ }^{3}$. With the advent of sequencing technologies, the wheat genome has been competitively sequenced several times ${ }^{4-6}$. The first reference-quality genome sequence with a comprehensive annotation was published by the IWGSC in August $2018^{7}$ for the accession Chinese Spring (CS). This assembly represents a tremendous resource for the scientific community and offers the promise of facilitating and accelerating breeding efforts.

More recently, fifteen genomes of hexaploid wheat have been published ${ }^{8}$ which represents a new step in the knowledge of the wheat model. Ten of these new wheat genomes have been assembled at the chromosome level, allowing for comparative analysis on a scale that was previously impossible. Although a valuable resource, these assemblies have been produced using short-read technologies and are therefore not up to the quality standard of current genomes $^{9-13}$. In 2017, an assembly of the CS genome using long-reads was produced ${ }^{5}$, although not annotated, highlighting the added-value of long-reads in such complex genomes. By accumulating long-read assemblies, the scientific community is now aware of the flaw in short-read strategies. Indeed they underestimate the repetitive content of the genome and more importantly can lack tandemly duplicated genes ${ }^{14,15}$. Several years ago, Pacific Biosciences (PACBIO) and Oxford Nanopore (ONT) sequencing technologies were commercialized with the promise to sequence long DNA fragments and revolutionize complex genome assemblies.

Here, we report the first hexaploid wheat genome based on ONT long-reads. We sequence the genome of a French variety (Renan) using the PromethION device and organize the assembled contigs at the chromosome scale using optical maps (BioNano Genomics, BNG) and Hi-C libraries (Arima Genomics, AG). This assembly has a contig N50 of $2.2 \mathrm{Mb}$, which is a 30 -fold improvement over existing chromosome-scale assemblies.

\section{Results}

\section{Genome sequencing and optical maps}

We sequenced genomic DNA using 20 ONT flow cells (2 MinION and 18 PromethION) which produced $12 \mathrm{M}$ reads representing $1.1 \mathrm{~Tb}$. All the reads were originally base called using the guppy 2.0 software, but given the improvement of guppy software during our project, we decided to call bases using a newer version of the guppy software (version 3.6 with High 
Accuracy setting). This dataset represented a coverage of $63 x$ of the hexaploid wheat genome and the read N50 was of $24.6 \mathrm{~kb}$. More importantly, we got $3.1 \mathrm{M}$ reads larger than $50 \mathrm{~kb}$ representing a 14x genome coverage (Table S1). In addition, we generated Illumina short-reads and long-range data for respectively polishing and organizing nanopore contigs. We produced an optical map using the Saphyr instrument commercialized by Bionano Genomics (BNG). High molecular weight DNA was extracted and labeled using the Direct Label and Stain Chemistry (DLS) with the DLE-1 enzyme. The DLE-1 optical map was assembled using proprietary tools provided by BNG and had a cumulative size of $14.9 \mathrm{~Gb}$ with an N50 of $37.5 \mathrm{Mb}$ (Table S2). Four Hi-C libraries from two biological replicates were prepared using the Arima Genomics protocol and sequenced on an Illumina sequencer to reach $537 \mathrm{~Gb}$ i.e., a depth of $35 \mathrm{x}$. We used a sample of 240 million read pairs ( $72 \mathrm{~Gb}, 5 \mathrm{x})$ to build a Hi-C map.

\section{Genome assembly}

Since the dataset was too large for many long-read assemblers, we sampled a $30 x$ coverage by selecting the longest reads (Table $\mathrm{S} 1$ ). This subset was assembled using multiple assembly tools dedicated to processing this large amount of data (Redbean ${ }^{16}$, SMARTdenovo ${ }^{17}$ and Flye ${ }^{18}$ ). SMARTdenovo is not among the fastest algorithms and has not been updated for several years, but since it can be easily parallelized, it remains an interesting choice for assembling large genomes. The overlap and consensus calculations were split into 60 chunks and each were run on a 32-core server and took about two days and ten hours respectively. In comparison, Redbean was able to generate an assembly after just seven days on a 64-core server with 3TB of memory while Flye needed 43 days on the same computer server. Surprisingly, the redbean assembly had a cumulative size two times higher than the expected genome size (29.6Gb vs $14.5 \mathrm{~Gb})$, a low contiguity and contained a large amount of short contigs. The SMARTdenovo and Flye assemblies were highly comparable, but Flye was the most contiguous (contigs $\mathrm{N} 50$ of $1.8 \mathrm{Mb}$ vs $1.1 \mathrm{Mb}$ ) and SMARTdenovo had a cumulative size closer to the expected one (14.1 Gb vs $13.0 \mathrm{~Gb}$, Table S3). Additionally, even though the assemblies were polished later, the raw SMARTdenovo assembly contained a higher number of complete BUSCO genes ( $83.0 \%$ vs $49.5 \%$ ) which indicates that its consensus module is more efficient.

The SMARTdenovo and Flye assemblies were successively polished using Racon ${ }^{19}$ and Medaka ${ }^{20}$ with long reads and Hapo- $\mathrm{G}^{21}$ with short reads. Polished contigs were validated and organized into scaffolds using the DLE-1 optical map and proprietary tools provided by BNG. As expected, due to its lower cumulative size, Flye scaffolds contained a larger 
proportion of unknown bases (851 $\mathrm{Mb}$ and $262 \mathrm{Mb})$. Based on this result, the assembly produced by SMARTdenovo ${ }^{17}$ was selected (Table S4). Local contig duplications (negative gaps) were resolved using $\mathrm{BiSCoT}^{22}$, which improved the contigs $\mathrm{N} 50$ from $1.2 \mathrm{Mb}$ up to 2.1 $\mathrm{Mb}$. Finally, the resulting assembly was polished one last time using Hapo- $\mathrm{G}^{21}$ with short reads. This led to 2,904 scaffolds (larger than $30 \mathrm{~kb}$ ) representing $14.26 \mathrm{~Gb}$ with a N50 of 48 $\mathrm{Mb}$ (79 scaffolds) and a maximum scaffold size of $254 \mathrm{Mb}$. Thus, the genome size is in the same range as all other available reference quality assemblies of T. aestivum: e.g. $14.29 \mathrm{~Gb}$ for $c v$. LongReach Lancer, $14.55 \mathrm{~Gb}$ for cv. Chinese Spring, and $14.96 \mathrm{~Gb}$ for cv. SY Mattis.

\section{Construction and validation of pseudomolecules}

We then guided the construction of the 21 chromosome sequences (i.e. pseudomolecules) based on collinearity with the CS (Chinese Spring) RefSeq Assembly v2.123. Given the complexity of this hexaploid genome, we established a dedicated approach in order to anchor each Renan scaffold based on similarity search against CS. To avoid problems due to multiple mappings, we selected a dataset of uniquely mappable sequences. Genes are not uniquely mappable since most of them are repeated as three homoeologous copies sharing on average $97 \%$ nucleotide identity. In addition, the gene density (1 gene every 130 $\mathrm{kb}$ on average) is too low to anchor small Renan scaffolds that do not carry genes. Thus, we used $150 \mathrm{bp}$ tags corresponding to the $5^{\prime}$ and $3^{\prime}$ junctions between a transposable element (TE) and its insertion site (75 bps on each side) which are called ISBP (Insertion Site-Based Polymorphism) markers and are highly abundant and uniquely mappable in the wheat genome ${ }^{24}$. We designed a dataset of 5.76 million ISBPs from CS assembly which represent 1 ISBP every $2.5 \mathrm{~kb}$. Their mapping enabled the anchoring of 2,566 scaffolds on 21 pseudomolecules representing $14.20 \mathrm{~Gb}$ (99\% of the assembly). We then used $\mathrm{Hi}-\mathrm{C}$ data to validate the assembly and to correct the mis-ordered and mis-oriented scaffolds. The $\mathrm{Hi}-\mathrm{C}$ map revealed only a few inconsistencies, demonstrating that the collinearity between CS and Renan was strong enough to guide the anchoring in a very accurate manner. The $\mathrm{Hi}-\mathrm{C}$ map-based curation led to the detection of 18 chimeric scaffolds that were split into 2 or 3 pieces and to the correction of the location and/or orientation of 198 scaffolds. The final assembly was composed of 21 pseudomolecules (Figure 1) with 338 unanchored scaffolds representing $61 \mathrm{Mb}$ only.

\section{Quality assessment of the assembly}

We first estimated the completeness and quality of the assembly by searching for the presence of known genes, i.e. the 107,891 High Confidence (HC) genes predicted in CS 
RefSeq v1.1. We used BLAST ${ }^{25}$ to search for the presence of each of the 491,456 exons larger than $30 \mathrm{bps}$ in the Renan scaffolds, and we considered only matches showing at least $90 \%$ identity over at least $90 \%$ query length. We found hits for $97.6 \%$ of the query exons with on average $99.3 \%$ identity, suggesting that the gene space is assembled at a high-quality level. The missing genes/exons would correspond, in most of the cases, to real presence/absence variations between CS and Renan while the nucleotide divergence between exons is only $0.7 \%$. It also demonstrated that homoeologous gene copies, sharing on average $97 \%$ identity $^{7}$ were not collapsed in the delivered Renan assembly. Indeed, $62 \%$ of the CS exons are strictly identical in Renan, showing that, even between a French and an Asian accession, SNPs in coding sequences are rare. It also confirmed that the Renan sequence quality is high even in homoeologous repeated sequences. We then assessed the assembly quality of the TE space by aligning the complete dataset of ISBP markers of CS onto the Renan assembly. We found that 94\% markers were conserved (at least $90 \%$ identity over $90 \%$ query length) i.e., present in the assembly, revealing that the TE space is extremely close to completeness. Indeed, $6 \%$ of missing markers is similar to the proportion of expected Presence-Absence variations (PAV) affecting TEs ${ }^{26}$.

Additionally, we aligned both short and long reads on the final assembly and examined the coverage in $100 \mathrm{~kb}$ windows. Interestingly, using short reads we found lower coverage of the D subgenome compared to the A and B subgenomes (Figures S1 and S2), which may indicate mapping issues with the short reads. We extracted the highly covered regions which may be candidate regions for homoelogous exchanges or may represent collapsed regions during the assembly. We found only 186 highly covered regions, representing $0.13 \%$ of the whole genome sequence, that were mainly localized on the A and B sugenomes (Figure 1) with few differences between short and long reads.

\section{Impact of the polishing}

Based on BUSCO and the alignment of the IBSP markers from the CS assembly, we monitored the evolution of the consensus quality through successive polishing iterations. As previously described, the SMARTdenovo consensus allowed the recovery of a greater number of complete BUSCO genes compared to that of Flye, which may be an indicator of its greater accuracy. However, the BUSCO score was still low (83\%) especially for a hexaploid genome, underlining the importance of polishing raw assemblies. Likewise, we were able to find $80.4 \%$ of the IBSP markers but only $7 \%$ were aligned without mismatch between the two genotypes (Table S5). When polished with long-reads, the BUSCO score reached $96.7 \%$ and $92.9 \%$ of the IBSP markers were retrieved (including $28.0 \%$ with perfect 
matches). The subsequent polishing step with short reads weakly decreased the BUSCO score (from $96.7 \%$ to $96.6 \%$ ), but the proportion of duplicated genes increased from $83.1 \%$ to $87.0 \%$ which is here wanted because in the case of a hexaploid genome most of the genes are in three copies. Moreover, the proportion of perfectly aligned ISBP markers drastically increased from $28.0 \%$ up to $58.9 \%$. Although the polishing with short reads weakly impacts the BUSCO conserved genes, the IBSP markers underline its importance in the case of long reads assemblies. Since ISBPs are unique tags sampling the whole genome, this analysis revealed that nucleotide errors were frequent before polishing, affecting half of the sample loci. Thus, we showed that the polishing steps were successful, even in this large and polyploid genome, and drastically improved the quality of the consensus.

\section{Recent improvement of the ONT technology}

Oxford Nanopore technology is evolving rapidly, and improvements to the base calling softwares are frequent, allowing old data to be analyzed with the aim of improving read accuracy and subsequent analysis. To measure the gain brought by each new version during this project, we analyzed a subset of ultra-long reads (longer than $100 \mathrm{~kb}$ ) with different basecallers or versions of the same basecaller: guppy 2.0, guppy 3.0.3 (High Accuracy mode), guppy 3.6 (High Accuracy mode) and the recent bonito v0.3.1. We observed a strong difference in accuracy, of around 7\%, between guppy 2.0 and the newer basecaller (bonito v0.3.1), representing the gain over the last two years (Figure 2A). This significant improvement could lead nanopore users to reanalyze their old sequencing data to improve the quality of their assemblies. Surprisingly, the identity percentage obtained on wheat is lower than what was obtained on yeast and human samples (Figure 2B). This difference can be explained by the fact that, first, the consensus of the wheat genome is not perfect and secondly, that basecallers are trained on a mixture which contains yeast and human data. Indeed, DNA modification patterns can differ between taxa, and read accuracy seems better when the model was trained on native DNA from the same species ${ }^{27}$. This huge difference between the read accuracy of yeast and wheat samples should motivate nanopore users to train basecaller models to their targeted species.

Additionally, we evaluated the improvement of a given assembly after a reanalysis of the sequencing data, and launched SMARTdenovo twice using ONT reads basecalled with guppy 3.3 and guppy 3.6 (Table S6). The accuracy of raw nanopore reads gained about $2 \%$ on average using guppy 3.6. We observed a reduction of the number of contigs of $19 \%$, and an improvement of the contig N50 of $26 \%$, which represents a substantial gain. Likewise, the 
cumulative size is slightly higher in the guppy 3.6 assembly, which may underline a smaller amount of collapsed repetitive regions (Table S7).

\section{Annotation of transposable elements and protein-coding genes}

We annotated TEs based on similarity search against our wheat-specific TE library ClariTeRep ${ }^{28}$ and raw results were then refined using CLARITE, a homemade program able to resolve prediction conflicts, merge adjacent features into a single complete element, and identify nested insertion patterns. We detected 3.9 million copies of TEs in the Renan genome assembly, representing $12.0 \mathrm{~Gb}$ i.e. $84 \%$ of the assembly size. The proportions of each superfamily were extremely similar to what has been described for $\mathrm{CS}^{29}$ (Table 2).

Gene annotation was achieved by, first, transferring genes predicted in CS RefSeq v2.1 by homology using the MAGATT pipeline ${ }^{23}$. This allowed us to accurately transfer 105,243 (out of 106,$801 ; 98 \%$ ) HC genes and 155,021 (out of 159,846; 97\%) Low Confidence genes. Such a transfer of genes predicted in another genotype (here CS) avoided genome-wide de novo gene prediction that may artificially lead to many differences between the annotations. We thus focused de novo predictions using TriAnnot ${ }^{30}$ only on the unannotated part of the genome, representing $8.5 \%$ of the $14.2 \mathrm{~Gb}$, after having masked transferred genes and predicted TEs. This method allowed us to predict 4,440 genes specific to Renan compared to CS i.e., $4 \%$ of the gene complement. This is consistent with the extent of structural variations affecting genomes of Triticeae ${ }^{26}$. Transfer of known genes, novel predictions, and manual curation (limited to storage protein encoding genes), led us to annotate 109,552 protein-coding genes on the Renan pseudomolecules.

\section{Comparison with existing hexaploid genome assemblies}

We compared our long-read assembly with 10 other available chromosome-scale assemblies of wheat genomes. Although the gene content was similar between the different assemblies, as expected, the assemblies based on short-read had a lower contiguity (contig $\mathrm{N} 50$ values lower than $100 \mathrm{~kb}$ compared to the $2 \mathrm{Mb}$ of the assembly of the Renan genome, Figure 3A-B). Logically, they also contained more gaps (around 40 times, Figure 3C). Interestingly, we found in general more gaps per $\mathrm{Mb}$ in the $\mathrm{D}$ subgenome compared to the $\mathrm{A}$ and $B$ subgenomes in Renan. This tendency is more pronounced in long-read assemblies (Figure S3). Chromosomes from the different assemblies had similar length except for the ArinaLrFor and the SY_Mattis variety in which a translocation has been previously described between chromosomes $5 B$ and $7 B^{8}$ (Figure $3 D$ ).

In addition, we generated dotplots between CS and Renan homeologous chromosomes and 
confirmed the strong collinearity between the two genomes (Figure 4). Whole chromosome alignments highlighted 16 large-scale inversions ( $>5 \mathrm{Mb}$; up to $118 \mathrm{Mb}$ ) on 10 chromosomes and 1 translocation of a ca. $45 \mathrm{Mb}$ segment on chromosome 4A. We performed the same comparisons with the 10 other available genomes of related varieties assembled at the pseudomolecule level (Supplementary Data 1). It showed that only 2 of these inversions are specific to Renan while the others are shared between several accessions. They correspond to regions of $23 \mathrm{Mb}$ on chr6B (position 398-421 Mb) and $10 \mathrm{Mb}$ on chr7B (position 267-277 $\mathrm{Mb})$.

\section{Comparative analysis of a storage protein coding gene cluster in T. aestivum}

Tandem duplications are an important mechanism in plant genome evolution and adaptation $^{31,32}$ but the assembly of tandemly duplicated gene clusters is difficult, especially with short-reads. In order to illustrate the gain brought by this optimized assembly process, we focused on an important locus on chromosome 1B known to carry multiple copies of storage protein and disease resistance genes ${ }^{33,34}$. Among them, the genes encoding omega-gliadins are not only duplicated in tandem, but are also composed of microsatellite DNA in their coding part, making them particularly hard to assemble properly from short reads. We compared orthologous regions harboring these genes between CS and Renan, spanning $1.58 \mathrm{Mb}$ and $2.32 \mathrm{Mb}$, respectively. The CS region was more fragmented with 81 gaps versus only 2 in Renan (Figures 5 ). The number of copies of omega-gliadin encoding genes was quite similar: 9 in CS and 10 in Renan. The most striking difference came from the completeness of the microsatellite motifs: 8 copies out of 9 contain $\mathrm{N}$ stretches in CS RefSeq v2.1, revealing that the microsatellite is usually too large to be fully assembled with short reads. In contrast, all 10 copies predicted in Renan were assembled completely. More generally, we mapped the corresponding proteins back to the locus and showed that it was better reconstructed in the Renan assembly, with a mean protein alignment length of $99 \%$ compared to $58 \%$ in CS.

\section{Discussion}

In this study, we showed that the recent improvement of the Oxford Nanopore technology, in terms of error rate and throughput, has opened up new perspectives in the age of long-read technologies. Indeed, the sequencing and assembly of complex genomes is now accessible to sequencing facilities. Additionally, the ability to sequence ultra-long reads using ONT devices is a real advantage over other long-read technologies, and the error rate that was previously a thorn in their side has been drastically reduced over the last year. By following 
basecallers evolution, we noticed that the gain when using recent basecaller is high and we guess this observation will encourage users to reprocess older data. However, this is not trivial and it requires sufficient computing resources. With all these recent improvements, it is clear that ONT now offers the ability to generate high-quality assemblies even in the case of complex genomes, like the hexaploid wheat.

Interestingly, we observed that the error rate of ONT data is highly organism dependent and that the training of basecaller has a significant impact on the overall quality of the reads. This is, in our opinion, an important fact because a large proportion of de novo assemblies now concern non-model organisms and users will have to address this limitation of current software. There are existing methods to train the basecaller on non-model species, but this can still be a big barrier, depending on the size of the dataset, for many end users. However, as highlighted in this study, the combination of long-reads and short-reads sequencing with polishing methods greatly improves the consensus sequence of a given genome assembly and these algorithms seem sufficient at least in coding regions.

Even though there are now several chromosome-scale assemblies of the hexaploid wheat genome, this assembly of the Renan variety based on long-reads will benefit biologists and geneticists as it offers a higher resolution. We demonstrated by examining an important loci containing prolamin and resistance genes that such regions are truly enhanced and contain very few gaps compared to assemblies based on short-reads. Additionally, unlike recent chromosome-scale assemblies, Renan's gene prediction is not only a projection of Chinese Spring gene models, but also includes de novo annotation which is of real benefit for the construction of pan genome (or pan annotation) or when cultivar-specific genes are examined. For all of these reasons, we believe this higher resolution assembly will benefit the wheat community and help breeding programs dedicated to the bread wheat genome.

\section{Methods}

\section{Plant material and DNA extraction}

Triticum aestivum cv. Renan seeds were provided by the INRAE Biological Resource Center on small grain cereals and grown for two weeks and a dark treatment was applied on the seedlings for two days before collecting leaf tissues. 
For the sequencing experiments, DNA was isolated from frozen leaves using QIAGEN Genomic-tips 100/G kit (Cat No./ID: 10243) and following the tissue protocol extraction. Briefly, $1 \mathrm{~g}$ of leaves were ground in liquid nitrogen with mortar and pestle. After $3 \mathrm{~h}$ of lysis and one centrifugation step, the DNA was immobilized on the column. After several washing steps, DNA is eluted from the column, then desalted and concentrated by alcohol precipitation. The DNA is resuspended in the TE buffer.

To generate the optical map, uHMW DNA were purified from 0.5 gram of very young fresh leaves according to the Bionano Prep Plant tissue DNA Isolation Base Protocol (30068 Bionano Genomics) with the following specifications and modifications. Briefly, the leaves were fixed using a fixing solution (Bionano Genomics) containing formaldehyde (Sigma-Aldrich) and then grinded in a homogenization buffer (Bionano Genomics) using a Tissue Ruptor grinder (Qiagen). Nuclei were washed and embedded in agarose plugs. After overnight proteinase $\mathrm{K}$ digestion in Lysis Buffer (Bionano Genomics) and one hour treatment with RNAse A (Qiagen), plugs were washed four times in 1x Wash Buffer (Bionano Genomics) and five times in 1x TE Buffer (ThermoFisher Scientific). Then, plugs were melted two minutes at $70^{\circ} \mathrm{C}$ and solubilized with $2 \mu \mathrm{L}$ of $0.5 \mathrm{U} / \mu \mathrm{L}$ AGARase enzyme (ThermoFisher Scientific) for 45 minutes at $43^{\circ} \mathrm{C}$. A dialysis step was performed in $1 \mathrm{x}$ TE Buffer (ThermoFisher Scientific) for 45 minutes to purify DNA from any residues. The DNA samples were quantified by using the Qubit dsDNA BR Assay (Invitrogen). Quality of megabase size DNA was validated by pulsed field gel electrophoresis (PFGE).

\section{Illumina Sequencing}

DNA $(1.5 \mu \mathrm{g})$ was sonicated using a Covaris E220 sonicator (Covaris, Woburn, MA, USA). Fragments $(1 \mu \mathrm{g})$ were end-repaired, 3'-adenylated and Illumina adapters (Bioo Scientific, Austin, TX, USA) were then added using the Kapa Hyper Prep Kit (KapaBiosystems, Wilmington, MA, USA). Ligation products were purified with AMPure XP beads (Beckman Coulter Genomics, Danvers, MA, USA). Libraries were then quantified by qPCR using the KAPA Library Quantification Kit for Illumina Libraries (KapaBiosystems), and library profiles were assessed using a DNA High Sensitivity LabChip kit on an Agilent Bioanalyzer (Agilent Technologies, Santa Clara, CA, USA). The library was sequenced on an Illumina NovaSeq instrument (Illumina, San Diego, CA, USA) using 150 base-length read chemistry in a paired-end mode. After the Illumina sequencing, an in-house quality control process was applied to the reads that passed the Illumina quality filters ${ }^{35}$. These trimming and removal steps were achieved using Fastxtend tools ${ }^{36}$. 


\section{Nanopore Sequencing}

Libraries were prepared according to the protocol Genomic DNA by ligation (SQK-LSK109 kit). Genomic DNA fragments $(1.5 \mu \mathrm{g})$ were repaired and 3'-adenylated with the NEBNext FFPE DNA Repair Mix and the NEBNext ${ }^{\circledR}$ Ultra ${ }^{\text {TM }}$ II End Repair/dA-Tailing Module (New England Biolabs, Ipswich, MA, USA). Sequencing adapters provided by Oxford Nanopore Technologies (Oxford Nanopore Technologies Ltd, Oxford, UK) were then ligated using the NEBNext Quick Ligation Module (NEB). After purification with AMPure XP beads (Beckmann Coulter, Brea, CA, USA), the library was mixed with the Sequencing Buffer (ONT) and the Loading Bead (ONT) and loaded on MinION or PromethION R9.4.1 flow cells. One PromethION run was performed with Genomic DNA purified with Short Read Eliminator kit (Circulomics, Baltimore, MD, USA) before the library preparation.

\section{Optical Maps}

Labeling and staining of the uHMW DNA were performed according to the Bionano Prep Direct Label and Stain (DLS) protocol (30206 - Bionano Genomics). Briefly, labeling was performed by incubating 750 ng genomic DNA with 1× DLE-1 Enzyme (Bionano Genomics) for 2 hours in the presence of $1 \times$ DL-Green (Bionano Genomics) and 1× DLE-1 Buffer (Bionano Genomics). Following proteinase $\mathrm{K}$ digestion and DL-Green cleanup, the DNA backbone was stained by mixing the labeled DNA with DNA Stain solution (Bionano Genomics) in presence of $1 \times$ Flow Buffer (Bionano Genomics) and 1× DTT (Bionano Genomics), and incubating overnight at room temperature. The DLS DNA concentration was measured with the Qubit dsDNA HS Assay (Invitrogen).

Labelled and stained DNA was loaded on Saphyr chips. Loading of the chips and running of the Bionano Genomics Saphyr System were all performed according to the Saphyr System User Guide (30247 - Bionano Genomics). Data processing was performed using the Bionano Genomics Access software.

A total of $4541 \mathrm{~Gb}$ data were generated. From this data, molecules with a size larger than $150 \mathrm{~kb}$ were filtered generating $1931 \mathrm{~Gb}$ of data. These filtered data, corresponding to $128 \mathrm{x}$ coverage of the Triticum aestivum cv. Renan consists of 7,810,298 molecules with an N50 of $237.5 \mathrm{~kb}$ and an average label density of $14.3 / 100 \mathrm{kbp}$. The filtered molecules were aligned using RefAligner with default parameters. It produced 1053 genome maps with a N50 of 37.5 Mbp for a total genome map length of $14946.8 \mathrm{Mbp}$. 


\section{Long reads genome assembly}

The 20 ONT runs were basecalled using two versions of guppy: 3.3 HAC and 3.6 HAC (Table S6). We monitored the gain of each guppy basecaller release and evaluated three different assemblers in the context of large genomes: Redbean ${ }^{16} \mathrm{v} 2.5$ (git commit $3 \mathrm{~d} 51 \mathrm{~d} 7 \mathrm{e}$ ), SMARTdenovo $^{17}$ (git commit 5cc1356) and Flye $^{18}$ v2.7 (git commit 5c12b69). All assemblers were launched using a subset of reads consisting of $30 \mathrm{X}$ of the longest reads (Table S3). Then, we selected one of the assemblies based not only on contiguity metrics such as N50 but also cumulative size, proportion of unknown bases. The Flye (longest reads) and SMARTdenovo (all reads) assemblies were very similar in terms of contiguity but we decided to keep the SMARTdenovo assembly as its cumulative size was higher. The SMARTdenovo assembler using the longest reads resulted in a contig N50 of $1.1 \mathrm{Mb}$ and a cumulative size of $14.07 \mathrm{~Gb}$. As nanopore reads contain systematic error in homopolymeric regions, we polished the consensus of the selected assembly with nanopore reads as input to the Racon (v1.3.2, git commit 5e2ecb7) and Medaka softwares. In addition, we polished the assembly two additional times using Illumina reads as input to the Hapo-G tool (v1.0, git commit).

\section{Long range genome assembly}

The Bionano Genomics scaffolding workflow (Bionano Solve version 3.5.1) was launched with the nanopore contigs and the Bionano map. We found in several cases that the nanopore contigs were overlapping (based on the optical map) and these overlaps were corrected using the BiSCoT software ${ }^{22}$ with default parameters. Finally, the consensus sequence was polished once more using Hapo-G and short reads, to ensure correction of duplicate regions that were collapsed (Table S4).

\section{Validation of the Triticum aestivum cv Renan assembly}

We used BLAST ${ }^{25}$ to search for the presence of $107,891 \mathrm{HC}$ genes from CS RefSeq $\mathrm{v} 1.1$ in the Renan genome sequence. We extracted the 491,456 individual exons larger than $30 \mathrm{bps}$ from this dataset and computed exon-by-exon BLAST in order to avoid spurious sliced alignments. An exon was considered present if it matched the Renan scaffolds with at least $90 \%$ identity over at least $90 \%$ of its length. We extracted all available ISBPs (150 bps each) from the CS RefSeq v1.1 and filtered out ISBPs containing Ns and those that do not map uniquely on the CS genome. This led to the design of a dataset containing 5,394,172 ISBPS which were aligned on the Renan scaffolds using BLAST. We considered an ISBP was conserved in Renan if it matched with at least $90 \%$ identity over $90 \%$ of its length. We used 
the same ISBP dataset to study the impact of polishing on error rate in the assembly while using BLAST and considering at least $90 \%$ identity over at least 145 aligned nucleotides.

\section{Anchoring of the Triticum aestivum cv Renan assembly}

We guided the construction of 21 Renan pseudomolecules based on collinearity with the CS RefSeq Assembly v2.1. For this, we used the positions of conserved ISBPs as anchors $(5,087,711$ ISBPs matching with $>=80 \%$ identity over $>=90 \%$ query overlap). This represented 357 ISBPs/Mb, meaning that even the smallest scaffolds (30 kb) carried generally more than 10 potential anchors. However, some ISBPs match at non-orthologous positions which create noise to precisely determine the order and orientation of some scaffolds. To overcome this issue, we considered ISBPs by pairs. Only pairs of adjacent ISBPs (i.e. separated by less than $50 \mathrm{~kb}$ on both CS and Renan genomes) were kept as valid anchors, allowing the filtering out of isolated mis-mapped ISBPs. Only scaffolds harboring at least $50 \%$ of valid ISBP pairs on a single chromosome were kept. The others were considered unanchored and they comprised the "chrUn". We calculated the median position of matching ISBP pairs along each CS chromosome for defining the order of the Renan scaffolds relative to each other. Their orientation was retrieved from the orientation of all matching ISBP pairs in CS following the majority rule. We thus built 21 pseudomolecules that were then corrected according to the $\mathrm{HiC}$ map as explained hereafter.

Two Hi-C biological replicates were prepared from ten-days plantlets of Triticum aestivum cv. Renan following the Arima Hi-C protocol (Arima Hi-C User Guide for Plant Tissues DOC A160106 v01). For each replicate, two libraries were constructed using the Kapa Hyper Prep kit (Roche) according to Arima's recommendation (Library Preparation using KAPA Hyper Prep Kit DOC A160108 v01). The technical replicates were then pooled and sent to Genewiz for sequencing on an Illumina HiSeq4000 (four lanes in total), reaching a 35x coverage. We mapped a sample of 240 million read pairs with BWA-MEM (Burrows-Wheeler Aligner, Heng $\mathrm{Li}, 2013)$ to the formerly built 21 pseudomolecules, filtered out for low quality, sorted, and deduplicated using the Juicer pipeline ${ }^{37}$. We produced a Hi-C map from the Juicer output by the candidate assembly visualizer mode of 3D-DNA pipeline ${ }^{38}$ and visualized it with the Juicebox Assembly Tools software. Based on abnormal frequency contacts signals revealing a lack of contiguity, scaffold-level modifications of order, orientation and/or chimeric scaffolds were identified in order to improve the assembly. In case of chimeric scaffolds, coordinates of resulting fragments were retrieved from the Juicebox Assembly Tools application but then recalculated to correspond precisely to the closest gap in the scaffold. Pseudomolecules were eventually rebuilt from initial scaffolds and new fragments while adding $100 \mathrm{~N}$ gaps 
between neighbor scaffolds. A final Hi-C map was built to validate the accuracy of the final assembly.

\section{Calculation of chromosome coverage}

Short and long-reads were aligned using minimap2 (with the following parameters '-I 17G -2 --sam-hit-only -a -x sr' and '-I $17 \mathrm{G}$-2 --sam-hit-only --secondary=no -a -x map-ont' respectively). Coverage of individual chromosomes was calculated in $100 \mathrm{~Kb}$ windows using mosdepth $^{39}$ (version 0.3.1) and the following parameters '--by 100000 -n -i 2 -Q 10 -m'. For each chromosome, $100 \mathrm{~Kb}$ windows with a coverage higher than two times the median coverage of the corresponding chromosome were tagged highly covered. Only genomic regions with at least five consecutives $100 \mathrm{~Kb}$ windows were kept and represented in Figure 1.

\section{Transposable elements annotation}

Transposable elements were annotated using CLARITE $^{28}$. Briefly, TEs were identified through a similarity search approach based on the ClariTeRep curated databank of repeated elements using RepeatMasker (www.repeatmasker.org) and modelled with the CLARITE program that was developed to resolve overlapping predictions, merge adjacent fragments into a single element when necessary, and identify patterns of nested insertions ${ }^{28}$.

\section{Gene prediction}

We used MAGATT pipeline (Marker Assisted Gene Annotation Transfer for Triticeae, https://forgemia.inra.fr/umr-gdec/magatt) to map the full set of 106,801 High Confidence and 159,848 Low Confidence genes predicted in Chinese Spring IWGSC RefSeq v2.1. The workflow implemented in this pipeline was described in Zhu et al. ${ }^{23}$. Briefly, it uses gene flanking ISBP markers in order to determine an interval that is predicted to contain the gene before homology-based annotation transfer, limiting problems due to multiple mapping. When the interval is identified, MAGATT uses BLAT ${ }^{40}$ to align the gene (UTRs, exons, and introns) sequence and recalculate all sub-features coordinates if the alignment is full-length and without indels. If the alignment is partial or contains indels, it runs GMAP $^{41}$ to perform spliced alignment of the candidate CDS inside the interval. If no ISBP-flanked interval was determined or if both BLAT and GMAP failed to transfer the gene, MAGATT runs GMAP against the whole genome, including the unanchored fraction of the Renan assembly. We kept the best hit considering a minimum identity of $70 \%$ and a minimum coverage of $70 \%$, with cross_species parameter enabled. 
We then masked the genome sequence based on mapped genes and predicted transposable elements coordinates using BEDTools ${ }^{42}$ mergeBed and maskfasta v2.27.1. Hence, we computed a de novo gene prediction on the unannotated part of the genome. We used TriAnnot ${ }^{30}$ to call genes based on a combination of evidence: de novo predictions of gene finders (FGeneSH, Augustus), similarity with known proteins in Poaceae, and similarity with transcribed sequences, as described previously ${ }^{7}$. For that purpose, we produced RNASeq data for Renan from 28 samples corresponding to 14 different organs/conditions in replicates: grains at four developmental stages $(100,250,500$, and 700 degree days) under heat stress and control conditions, stems at two developmental stages, leaves at three stages, and roots at one stage), representing on average 78.8 million read-pairs per sample i.e., 2.2 billion read-pairs in total. We mapped reads with hisat $2^{43}$ v2.0.5, called 277,505

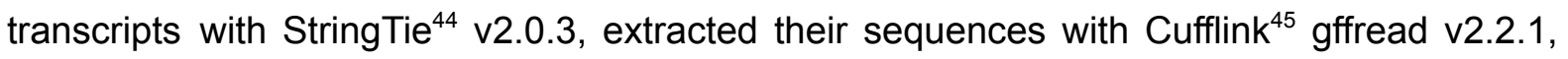
and provided this resource as input to TriAnnot. We optimized TriAnnot workflow to ensure a flawless use on a cloud-based hpc cluster (10 nodes with 32 CPUs/128GB RAM each and shared file system) using the laaS Openstack infrastructure from the UCA Mesocentre. Gene models were then filtered as follows: we discarded gene models that shared strong identity (>=92\% identity, >=95\% query coverage) with an unannotated region of the Chinese Spring RefSeq v2.1, considered as doubtful predictions. We then kept all predictions that matched RNASeq-derived transcripts (>=99\% identity, >=70\% query and subject coverage). For those that did not show evidence of transcription, we kept gene models sharing protein similarity ( $>=40 \%$ identity, $>=50 \%$ query and subject coverage) with a Poaceae protein having a putative function (filtering out based on terms "unknown", "uncharacterized", and "predicted protein").

\section{Comparison of genome assemblies}

Genome assemblies were downloaded from https://webblast.ipk-gatersleben.de/downloads. Contigs were extracted by splitting input sequences at each $\mathrm{N}$ and standard metrics were computed. Gene completion metrics were calculated using BUSCO v5.0 and version 10 of the poales geneset which contains 4896 genes.

We built dotplots between Renan, CS and 10 other reference quality genomes (ArinaLrFor, CDC Landmark, CDC Stanley, Jagger, Julius LongReach Lancer, Mace, Norin61, SY Mattis, spelta PI190962) by using orthologous positions of conserved ISBPs (1 ISBP every $2.5 \mathrm{~kb}$ on average) identified by mapping them with BWA-MEM (maximum 2 mismatches, 100\% coverage and minimal mapping quality of 30 ). 


\section{Comparison of a storage protein coding gene cluster}

We performed manual curation of the gene models encoding storage proteins predicted in Renan. Protein sequences of prolamin and resistance genes ${ }^{33}$ from a $1 \mathrm{~B}$ chromosome locus were downloaded and aligned to the CS and Renan genomes using BLAT ${ }^{40}$ with default parameters. Draft alignments were refined by aligning the given protein sequence and the genomic region defined by the blat alignment using Genewise with default parameters. Resulting alignments were filtered in order to conserve only the best match for each position by keeping only the highest-scoring alignment and the genomic region containing the gene cluster was extracted. Then, we used the jcvi suite ${ }^{46}$ with the mcscan pipeline to find synteny blocks between both genomes. First, we used the "jcvi.compara.catalog" command to find orthologs and then the "jcvi.compara.synteny mcscan" with "--iter=1" command to extract synteny blocks. Finally, we generated the figure with the "jcvi.graphics.synteny" command and manually edited the generated svg file to improve the quality of the resulting image by changing gene colors, incorporating gaps and renaming genes. Moreover, to make the figure clearer, we artificially reduced the intergenic space by $95 \%$ so that gene structures appear bigger. The omega gene cluster representation figure was generated by using DnaFeaturesViewer ${ }^{47}$ with coordinates of features generated by the mcscan pipeline used previously.

\section{Additional files}

All the supporting data are included in two additional files: (a) A supplementary file which contains Supplementary Tables 1-7 and Supplementary Figures 1-3; (b) A supplementary file which contains dotplots of the 21 chromosomes of Renan with other wheat genome assemblies.

\section{Acknowledgements}

This work was supported by the Genoscope, the Commissariat à l'Énergie Atomique et aux Énergies Alternatives (CEA) and France Génomique (ANR-10-INBS-09-08). The biological material (i.e. plant production, sample management, DNA and RNA extractions performed by Caroline Pont and Cécile Huneau at GDEC) have been obtained in the framework of the France Génomique WheatOMICS project (2017-2021) coordinated by Jérôme Salse. The authors are grateful to Oxford Nanopore Technologies Ltd for providing early access to the PromethION device through the PEAP, and we thank the staff of Oxford Nanopore 
Technologies Ltd for technical help. We are grateful to the Mésocentre Clermont Auvergne University and/or AuBi platform for providing help and/or computing and/or storage resources.

\section{Availability of supporting data}

The Illumina and PromethION sequencing data and the Bionano optical map are available in the European Nucleotide Archive under the following project PRJEB46515. The genome assembly and gene predictions are freely available from the Genoscope website http://www.genoscope.cns.fr/plants/.

\section{Competing interests}

The authors declare that they have no competing interests. JMA received travel and accommodation expenses to speak at Oxford Nanopore Technologies conferences. JMA and $\mathrm{CB}$ received accommodation expenses to speak at Bionano Genomics user meetings.

\section{Funding}

This work was supported by the Genoscope, the Commissariat à l'Énergie Atomique et aux Énergies Alternatives (CEA) and France Génomique (ANR-10-INBS-09-08).

\section{Author's contributions}

SA, ID and $A B$ extracted the sequenced DNA and generated the optical map. KL and AA optimized and performed the nanopore and Illumina sequencing. NP, EP and MR generated the Hi-C libraries and sequences. JMA, SE, BI, CM, PLZ, CB, HR, PL, DG and FC performed the bioinformatic analyses. JMA, SE, BI, CM, PLZ, CB, CC, HR, PL and FC wrote the article. JMA, PW and FC supervised the study. 
Table 1: Comparison of Triticum aestivum L. genome assemblies.

\begin{tabular}{|c|c|c|c|}
\hline & \\
\hline & & $\begin{array}{l}\text { Renan } \\
\text { This study }\end{array}$ & $\begin{array}{c}\text { Chinese Spring } \\
\text { IWGSC }^{7}\end{array}$ \\
\hline \multicolumn{2}{|c|}{ Number of contigs } & 12,608 & 693,050 \\
\hline \multicolumn{2}{|c|}{ Cumulative size (bp) } & $13,943,021,299$ & $14,271,578,887$ \\
\hline \multirow{2}{*}{\multicolumn{2}{|c|}{$\begin{array}{l}\text { N50 (bp) } \\
\text { L50 }\end{array}$}} & $2,164,453$ & 51,835 \\
\hline & & 1,946 & 81,466 \\
\hline \multirow{2}{*}{\multicolumn{2}{|c|}{$\begin{array}{l}\text { N90 (bp) } \\
\text { L90 }\end{array}$}} & 607,045 & 11,621 \\
\hline & & 6,582 & 295,310 \\
\hline \multicolumn{2}{|c|}{ Longest contig (bp) } & $15,116,687$ & 580,542 \\
\hline \multicolumn{2}{|c|}{ Number of chromosomes } & 21 & 21 \\
\hline \multicolumn{2}{|c|}{ Cumulative size (bp) } & $14,195,643,615$ & $14,547,261,565$ \\
\hline \multicolumn{2}{|l|}{ N50 (bp) } & $703,299,328$ & $709,773,760$ \\
\hline \multicolumn{2}{|l|}{ L50 } & 10 & 10 \\
\hline \multicolumn{2}{|l|}{ N90 (bp) } & $520,815,552$ & $509,857,056$ \\
\hline \multicolumn{2}{|l|}{ L90 } & 19 & 19 \\
\hline \multicolumn{2}{|c|}{ Longest (bp) } & $854,463,248$ & $830,829,764$ \\
\hline \multicolumn{2}{|l|}{$\%$ of $\mathrm{N}$} & $1.78 \%$ & $1.90 \%$ \\
\hline \multirow{4}{*}{$\begin{array}{l}\text { BUSCO } \\
(N=4,896)\end{array}$} & Complete & $99.1 \%$ & $99.3 \%$ \\
\hline & Duplicated & $94.7 \%$ & $96.0 \%$ \\
\hline & Fragmented & $0.1 \%$ & $0.1 \%$ \\
\hline & Missing & $0.8 \%$ & $0.6 \%$ \\
\hline \multicolumn{2}{|c|}{ Number of genes } & 109,552 & 107,891 \\
\hline \multicolumn{2}{|c|}{ Average number of exons } & 5.10 & 5.33 \\
\hline \multirow{4}{*}{$\begin{array}{l}\text { BUSCO } \\
(N=4,896)\end{array}$} & Complete & $99.1 \%$ & $99.5 \%$ \\
\hline & Duplicated & $94.6 \%$ & $98.2 \%$ \\
\hline & Fragmented & $0.2 \%$ & $0.1 \%$ \\
\hline & Missing & $0.7 \%$ & $0.4 \%$ \\
\hline
\end{tabular}


Table 2: TE classes proportions in Chinese Spring and Renan genome assemblies.

\begin{tabular}{|c|c|c|c|}
\hline & $\begin{array}{l}\text { Chinese Spring } \\
\text { RefSeq_v1.0 } \\
\text { from Zhu et al }\left.\right|^{23}\end{array}$ & $\begin{array}{l}\text { Chinese Spring } \\
\text { RefSeq_v2.1 } \\
\text { from Zhu et al }\left.\right|^{23}\end{array}$ & Renan RefSeq_v2.0 \\
\hline Genome size (bp) & $14,066,280,851$ & $14,225,829,371$ & $14,195,643,615$ \\
\hline $\mathrm{TE}(\mathrm{bp})$ & $11,921,309,743$ & $12,092,094,168$ & $11,967,447,100$ \\
\hline TE (\%) & 84.7 & 85.0 & 84.3 \\
\hline $\begin{array}{l}\text { Class I } \\
\text { (Retrotransposons) }\end{array}$ & 67.6 & 66.9 & 66.6 \\
\hline Gypsy (RLG) & 46.7 & 46.1 & 45.8 \\
\hline Copia (RLC) & 16.7 & 16.5 & 16.5 \\
\hline $\begin{array}{r}\text { Unclassified LTR } \\
\text { retrotransposons }(\mathrm{RLX})\end{array}$ & 3.24 & 3.3 & 3.2 \\
\hline LINE (RIX) & 0.9 & 1.1 & 1.1 \\
\hline SINE (SIX) & 0.01 & 0.01 & 0.01 \\
\hline $\begin{array}{l}\text { Class II (DNA } \\
\text { transposons)- Subclass } 1\end{array}$ & 16.5 & 17.0 & 16.9 \\
\hline CACTA (DTC) & 15.5 & 15.9 & 15.8 \\
\hline Mutator (DTM) & 0.38 & 0.44 & 0.44 \\
\hline $\begin{array}{r}\text { Unclassified DNA } \\
\text { transposons with TIR } \\
(\text { DTX) }\end{array}$ & 0.21 & 0.24 & 0.24 \\
\hline Harbinger (DTH) & 0.16 & 0.18 & 0.18 \\
\hline Mariner (DTT) & 0.16 & 0.17 & 0.17 \\
\hline $\begin{array}{r}\text { Unclassified DNA } \\
\text { transposons (DXX) }\end{array}$ & 0.06 & 0.06 & 0.06 \\
\hline hAT (DTA) & 0.006 & 0.009 & 0.009 \\
\hline Helitrons (DHH) & 0.004 & 0.01 & 0.01 \\
\hline Unclassified TE (XXX) & 0.68 & 0.95 & 0.82 \\
\hline
\end{tabular}


bioRxiv preprint doi: https://doi.org/10.1101/2021.08.24.457458; this version posted August 24, 2021. The copyright holder for this preprint

(which was not certified by peer review) is the author/funder, who has granted bioRxiv a license to display the preprint in perpetuity. It is made available under aCC-BY-NC 4.0 International license.

Figure 1. Genome overview of the 21 chromosomes of hexaploid T. aestivum Renan (the 7 A chromosomes are in blue, the $7 \mathrm{~B}$ chromosomes in orange and the $7 \mathrm{D}$ chromosomes in green). From inner to outer track: (i) Gene density, (ii) Density of CACTA (DNA transposon) elements, (iii) Density of Copia elements, (iv) Density of Gypsy elements, (v) dots represent highly covered regions (candidate regions of homoeologous exchanges or collapsed regions) with illumina reads (in red) and nanopore reads (in blue), (vi) Density of gaps. All densities are calculated in 1-Mb windows; blue and red colors in density plots indicate lower and higher values, respectively.

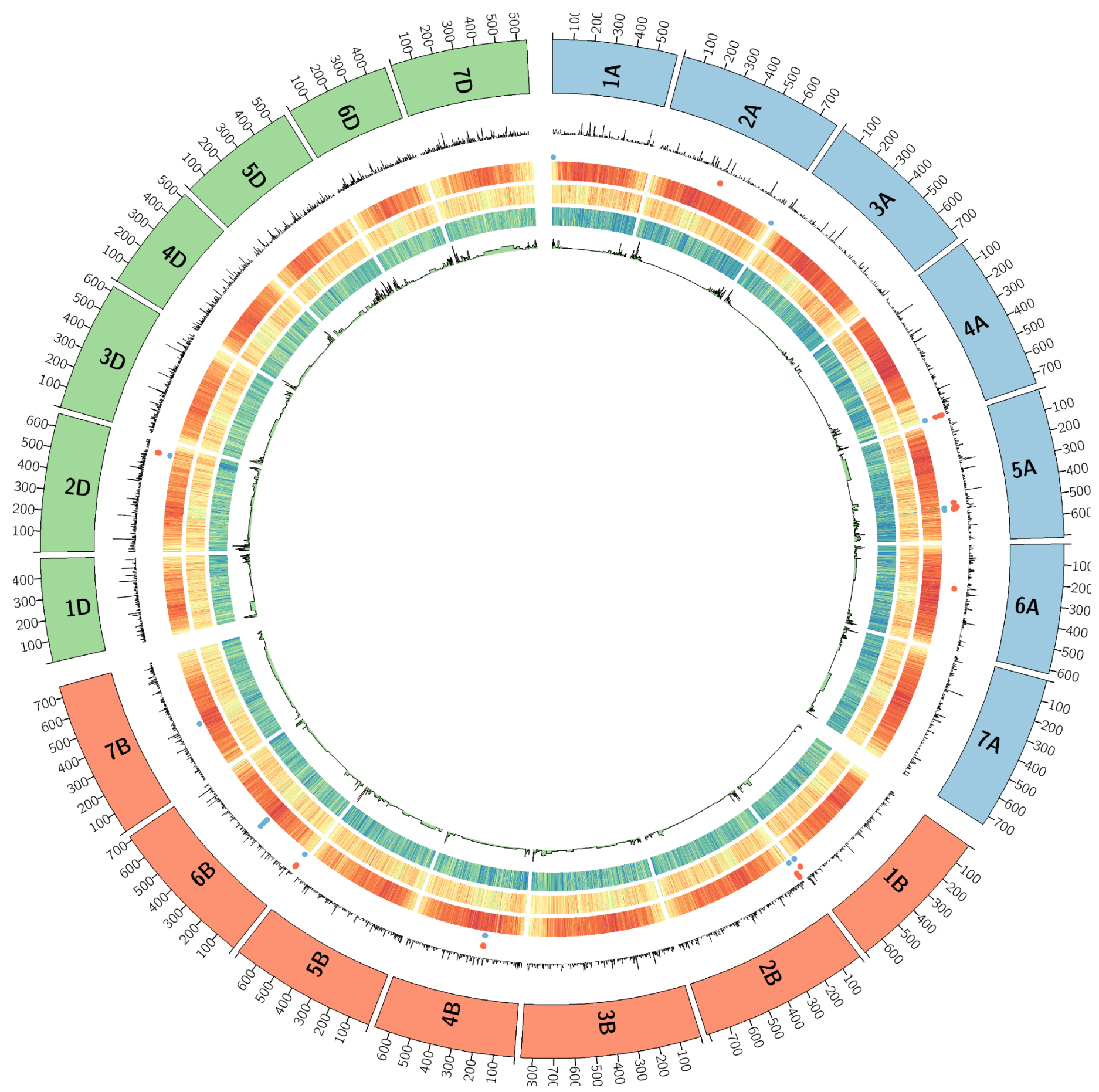


bioRxiv preprint doi: https://doi.org/10.1101/2021.08.24.457458; this version posted August 24, 2021. The copyright holder for this preprint

(which was not certified by peer review) is the author/funder, who has granted bioRxiv a license to display the preprint in perpetuity. It is made available under aCC-BY-NC 4.0 International license.

Figure 2. Comparison of the accuracy of different ONT basecallers. A. ONT reads from a yeast sample. B. ONT ultra-long reads $(>100 \mathrm{~kb})$ from a wheat sample.

A.

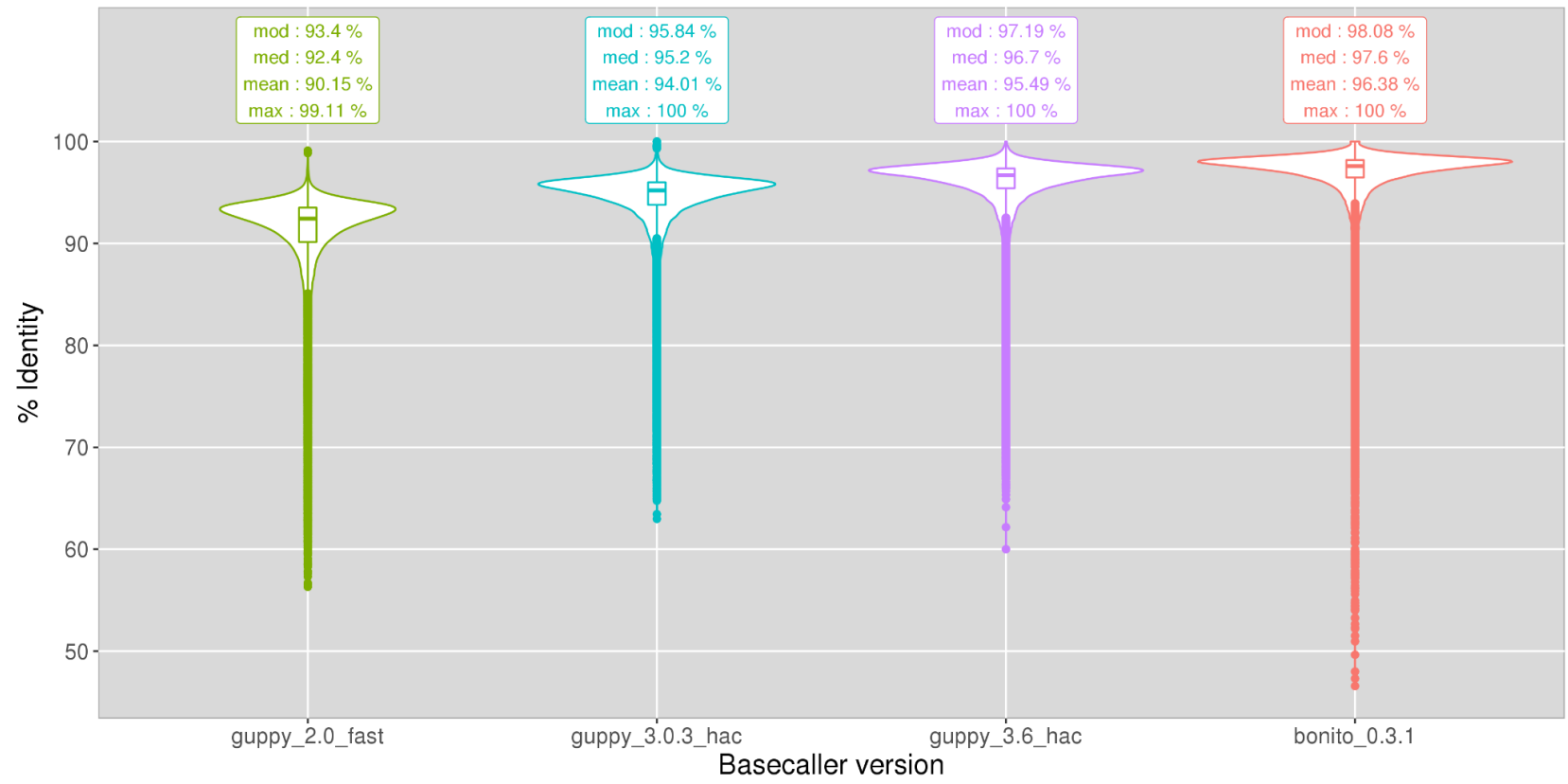

B.

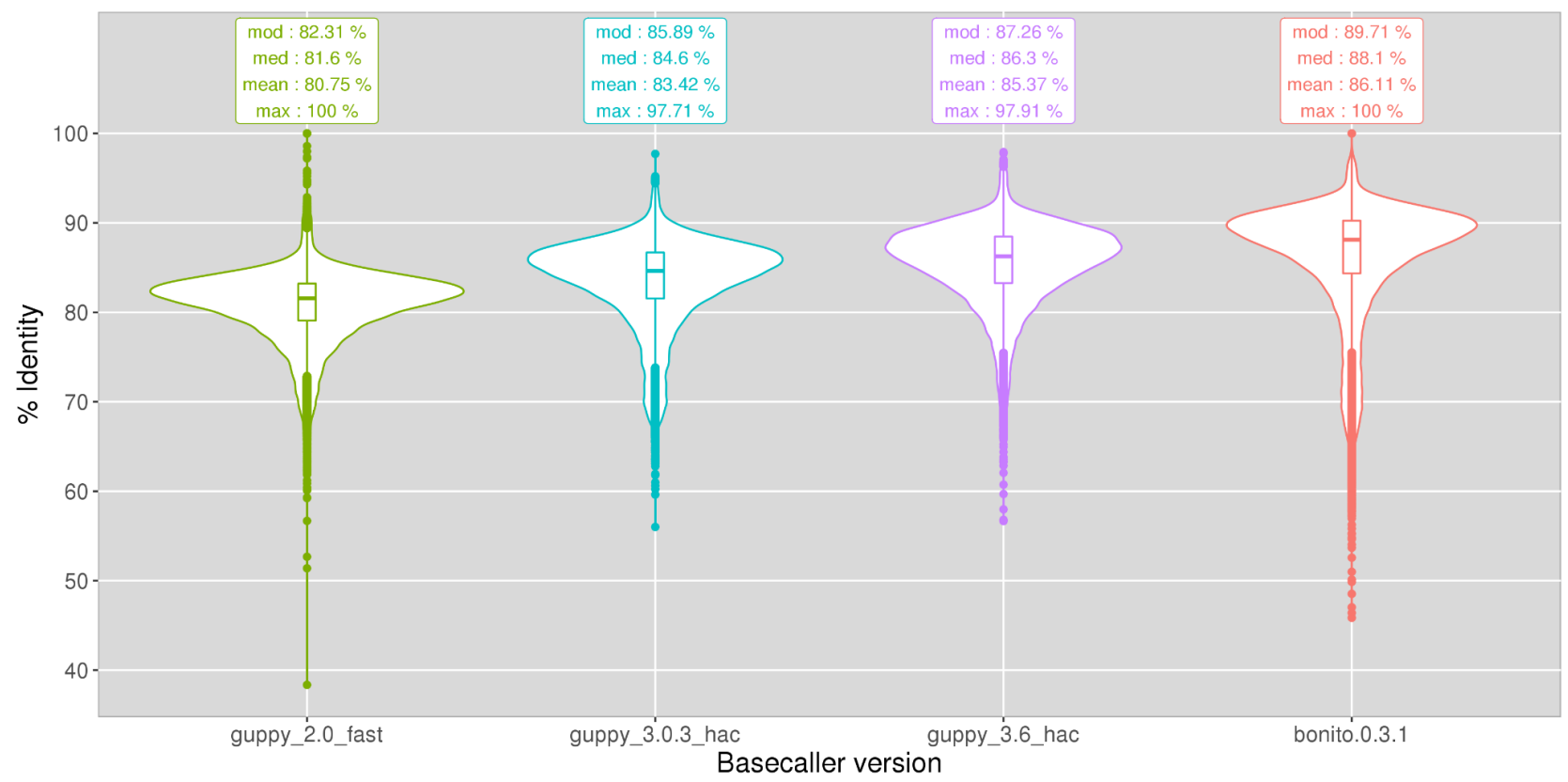


bioRxiv preprint doi: https://doi.org/10.1101/2021.08.24.457458; this version posted August 24,2021 . The copyright holder for this preprint

(which was not certified by peer review) is the author/funder, who has granted bioRxiv a license to display the preprint in perpetuity. It is made available under aCC-BY-NC 4.0 International license.

Figure 3. Comparison of existing hexaploid genome assemblies A. contig N50 values. B. Complete BUSCO genes found in each assembly. C. Number of gaps in each chromosome. D. chromosome length.

A

Contig N50 (Mb)

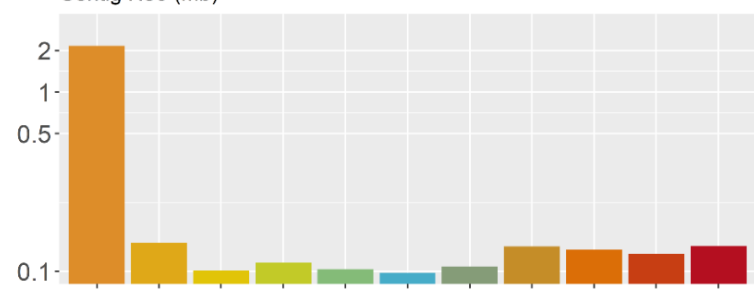

B
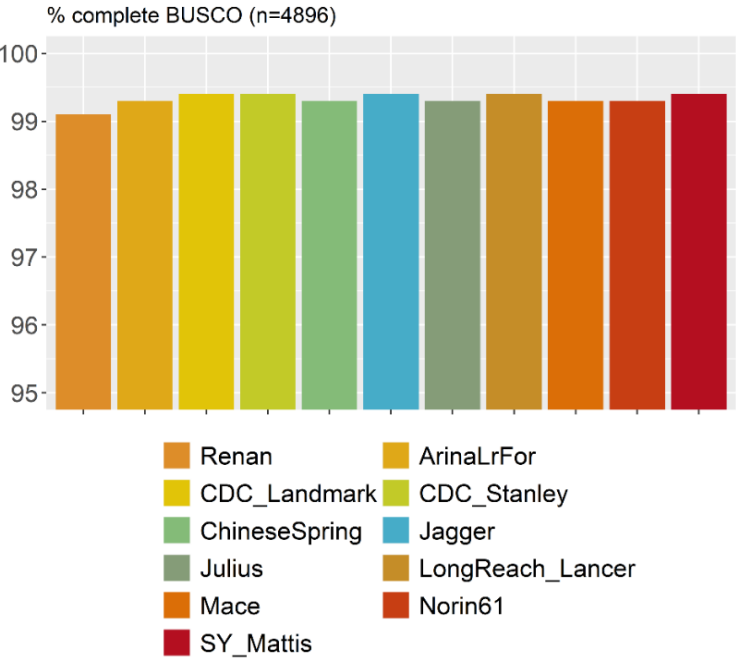

C

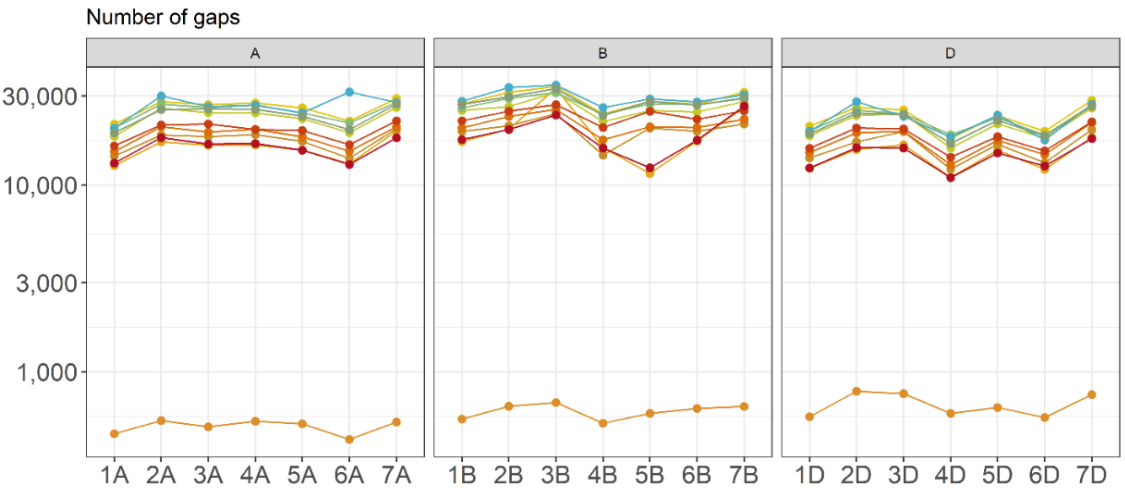

D

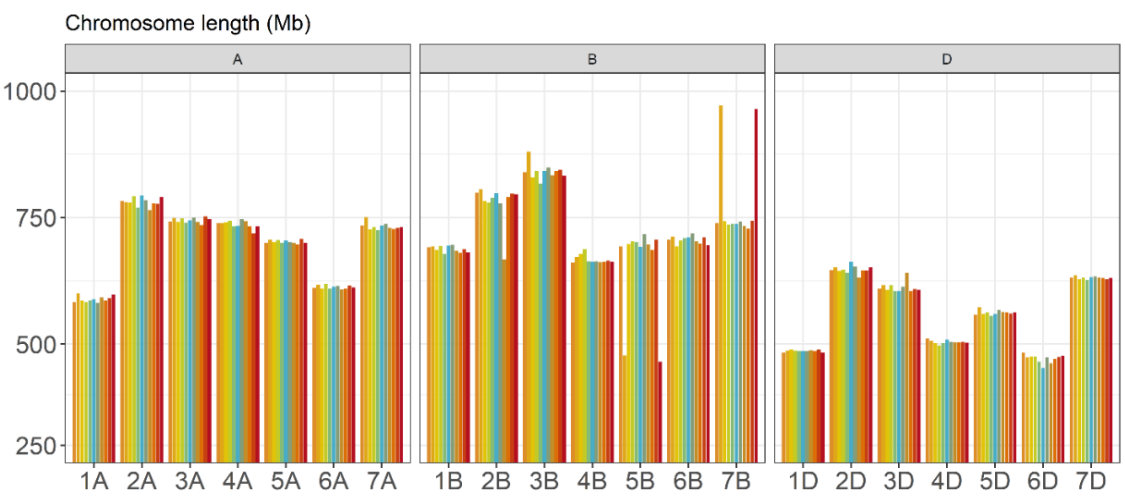


Figure 4. Dotplot comparisons of the 21 chromosomes of Renan ( $y$ axis) with the Chinese Spring RefSeq v2.1 assembly (x axis).
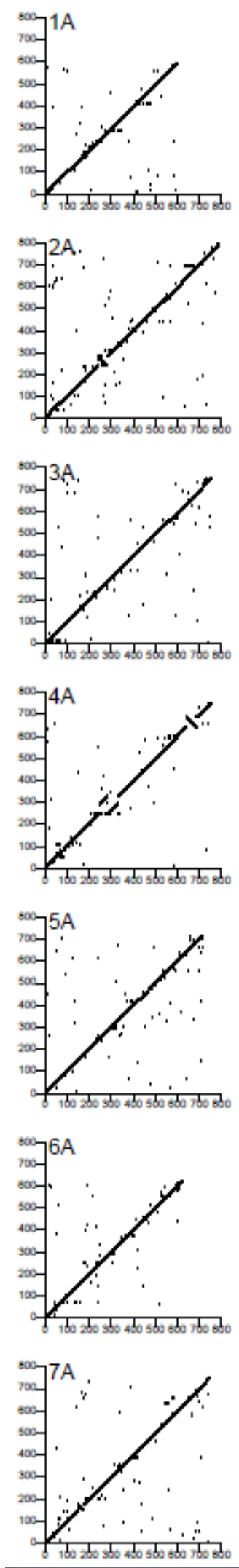
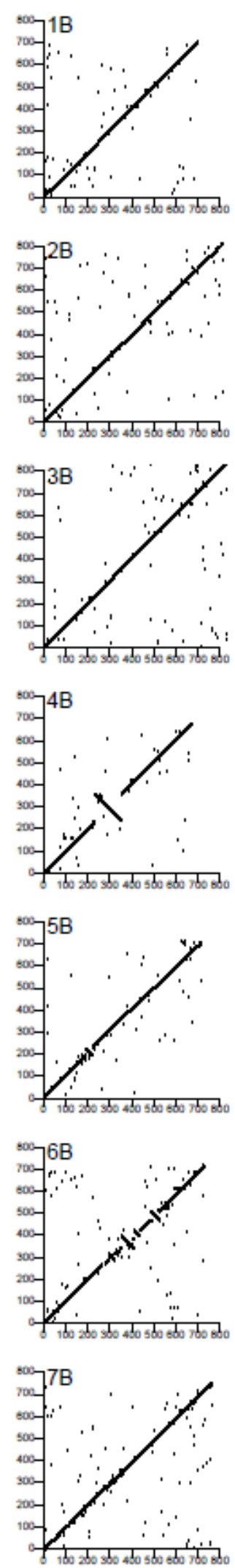
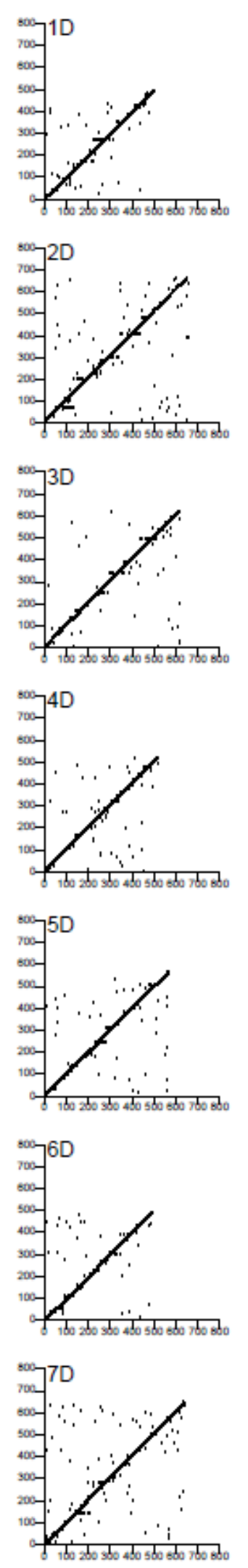
Figure 5. Comparative view of an important locus on chromosome $1 \mathrm{~B}$ containing prolamin and resistance genes, tandemly duplicated. a. Representation of the region with gaps and genes on the two assemblies of Renan and CS. b. Zoomed view on the omega gliadin gene cluster c. Proportion of the length of the proteins that were aligned in the genomic region of Renan and CS.

a.

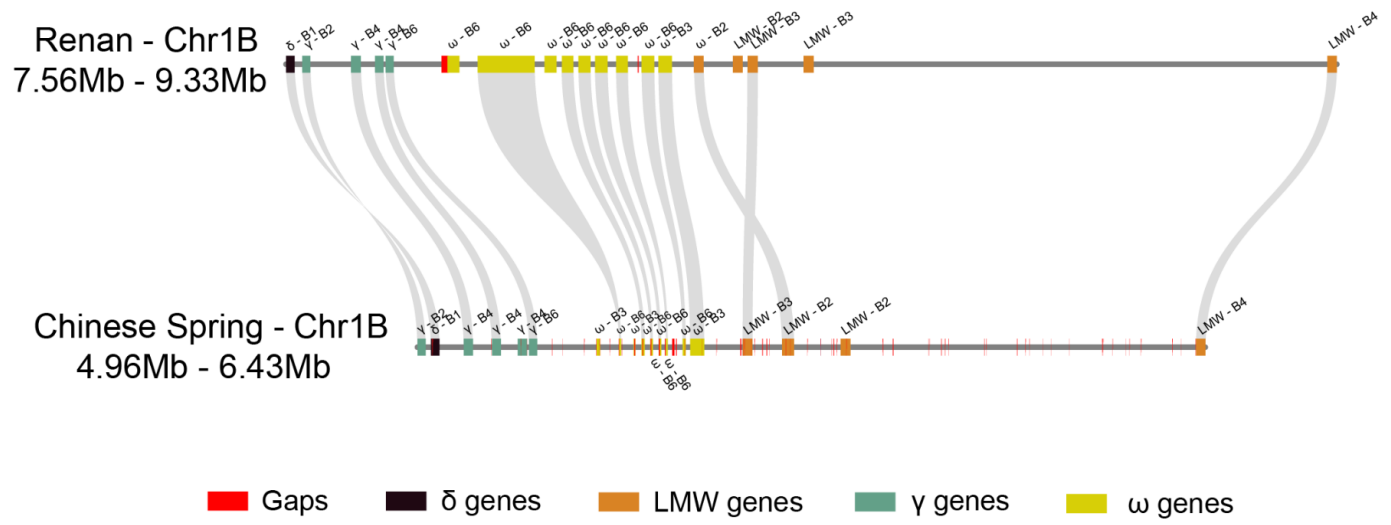

b.

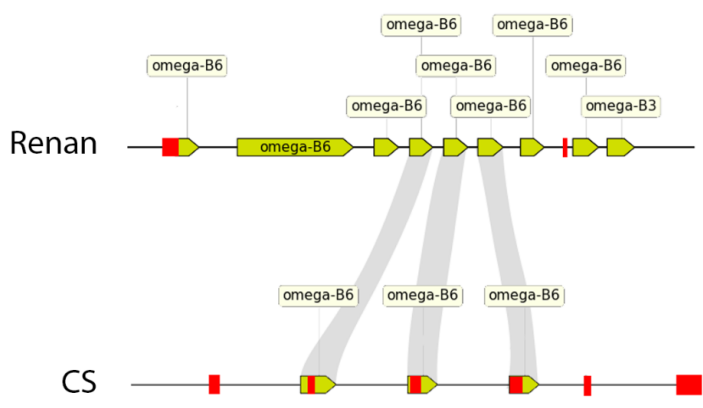

C.

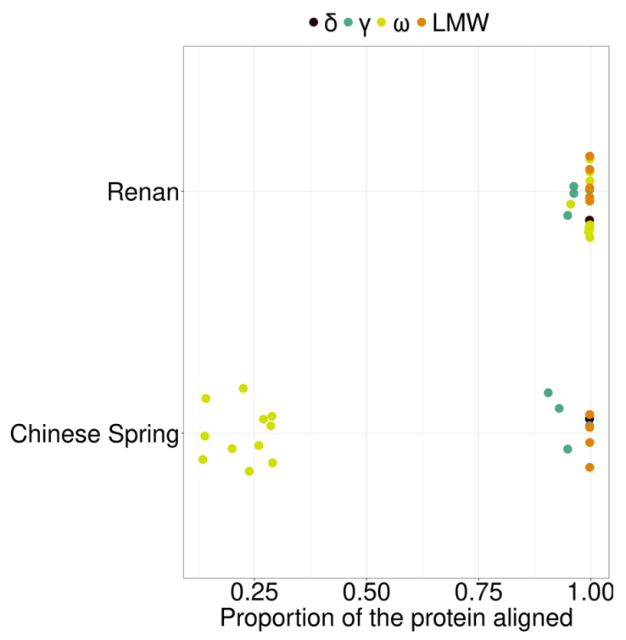




\section{References}

1. Dubcovsky, J. \& Dvorak, J. Genome Plasticity a Key Factor in the Success of Polyploid Wheat Under Domestication. Science 316, 1862-1866 (2007).

2. Marcussen, T. et al. Ancient hybridizations among the ancestral genomes of bread wheat. Science 345, 1250092 (2014).

3. Guan, J. et al. The Battle to Sequence the Bread Wheat Genome: A Tale of the Three Kingdoms. Genomics Proteomics Bioinformatics 18, 221-229 (2020).

4. Chapman, J. A. et al. A whole-genome shotgun approach for assembling and anchoring the hexaploid bread wheat genome. Genome Biol. 16, 26 (2015).

5. Zimin, A. V. et al. The first near-complete assembly of the hexaploid bread wheat genome, Triticum aestivum. GigaScience 6, 1-7 (2017).

6. Clavijo, B. J. et al. An improved assembly and annotation of the allohexaploid wheat genome identifies complete families of agronomic genes and provides genomic evidence for chromosomal translocations. Genome Res. 27, 885-896 (2017).

7. Consortium (IWGSC), T. I. W. G. S. et al. Shifting the limits in wheat research and breeding using a fully annotated reference genome. Science 361, (2018).

8. Walkowiak, S. et al. Multiple wheat genomes reveal global variation in modern breeding. Nature 588, 277-283 (2020).

9. Miga, K. H. et al. Telomere-to-telomere assembly of a complete human X chromosome. Nature 585, 79-84 (2020).

10. Belser, C. et al. Chromosome-scale assemblies of plant genomes using nanopore long reads and optical maps. Nat. Plants 4, 879-887 (2018).

11. Rousseau-Gueutin, M. et al. Long-read assembly of the Brassica napus reference genome Darmor-bzh. GigaScience 9, (2020).

12. Li, G. et al. A high-quality genome assembly highlights rye genomic characteristics and 
agronomically important genes. Nat. Genet. 53, 574-584 (2021).

13. Liu, J. et al. Gapless assembly of maize chromosomes using long-read technologies. Genome Biol. 21, 121 (2020).

14. Tørresen, O. K. et al. Tandem repeats lead to sequence assembly errors and impose multi-level challenges for genome and protein databases. Nucleic Acids Res. 47, 10994-11006 (2019).

15. Li, C. et al. Long-read sequencing reveals genomic structural variations that underlie creation of quality protein maize. Nat. Commun. 11, 1-11 (2020).

16. Ruan, J. \& Li, H. Fast and accurate long-read assembly with wtdbg2. Nat. Methods 17, 155-158 (2020).

17. Liu, H., Wu, S., Li, A. \& Ruan, J. SMARTdenovo: a de novo assembler using long noisy reads. Gigabyte 2021, 1-9 (2021).

18. Kolmogorov, M., Yuan, J., Lin, Y. \& Pevzner, P. A. Assembly of long, error-prone reads using repeat graphs. Nat. Biotechnol. 37, 540-546 (2019).

19. Vaser, R., Sović, I., Nagarajan, N. \& Šikić, M. Fast and accurate de novo genome assembly from long uncorrected reads. Genome Res. 27, 737-746 (2017).

20. Medaka : https://github.com/nanoporetech/medaka. (Oxford Nanopore Technologies, 2021).

21. Aury, J.-M. \& Istace, B. Hapo-G, haplotype-aware polishing of genome assemblies with accurate reads. NAR Genomics Bioinforma. 3, (2021).

22. Istace, B., Belser, C. \& Aury, J.-M. BiSCoT: improving large eukaryotic genome assemblies with optical maps. PeerJ 8, e10150 (2020).

23. Zhu, T. et al. Optical maps refine the bread wheat Triticum aestivum cv. Chinese Spring genome assembly. Plant J. n/a,.

24. Rimbert, H. et al. High throughput SNP discovery and genotyping in hexaploid wheat. PLOS ONE 13, e0186329 (2018). 
25. Altschul, S. F., Gish, W., Miller, W., Myers, E. W. \& Lipman, D. J. Basic local alignment search tool. J. Mol. Biol. 215, 403-410 (1990).

26. De Oliveira, R. et al. Structural Variations Affecting Genes and Transposable Elements of Chromosome 3B in Wheats. Front. Genet. 11, (2020).

27. Wick, R. R., Judd, L. M. \& Holt, K. E. Performance of neural network basecalling tools for Oxford Nanopore sequencing. Genome Biol. 20, 129 (2019).

28. Daron, J. et al. Organization and evolution of transposable elements along the bread wheat chromosome 3B. Genome Biol. 15, 546 (2014).

29. Wicker, T. et al. Impact of transposable elements on genome structure and evolution in bread wheat. Genome Biol. 19, 103 (2018).

30. Leroy, P. et al. TriAnnot: A Versatile and High Performance Pipeline for the Automated Annotation of Plant Genomes. Front. Plant Sci. 0, (2012).

31. Kondrashov, F. A. Gene duplication as a mechanism of genomic adaptation to a changing environment. Proc. R. Soc. B Biol. Sci. 279, 5048-5057 (2012).

32. Panchy, N., Lehti-Shiu, M. \& Shiu, S.-H. Evolution of Gene Duplication in Plants. Plant Physiol. 171, 2294-2316 (2016).

33. Huo, N. et al. Gene Duplication and Evolution Dynamics in the Homeologous Regions Harboring Multiple Prolamin and Resistance Gene Families in Hexaploid Wheat. Front. Plant Sci. 9, (2018).

34. Xu, J.-H. \& Messing, J. Organization of the prolamin gene family provides insight into the evolution of the maize genome and gene duplications in grass species. Proc. Natl. Acad. Sci. U. S. A. 105, 14330-14335 (2008).

35. Alberti, A. et al. Viral to metazoan marine plankton nucleotide sequences from the Tara Oceans expedition. Sci. Data 4, 170093 (2017).

36. Engelen S, Aury JM. fastxtend. https://www.genoscope.cns.fr/externe/fastxtend/.

37. Durand, N. C. et al. Juicer Provides a One-Click System for Analyzing Loop-Resolution 
Hi-C Experiments. Cell Syst. 3, 95-98 (2016).

38. Dudchenko, O. et al. De novo assembly of the Aedes aegypti genome using $\mathrm{Hi}-\mathrm{C}$ yields chromosome-length scaffolds. Science 356, 92-95 (2017).

39. Pedersen, B. S. \& Quinlan, A. R. Mosdepth: quick coverage calculation for genomes and exomes. Bioinforma. Oxf. Engl. 34, 867-868 (2018).

40. Kent, W. J. BLAT-The BLAST-Like Alignment Tool. Genome Res. 12, 656-664 (2002).

41. Wu, T. D. \& Watanabe, C. K. GMAP: a genomic mapping and alignment program for mRNA and EST sequences. Bioinformatics 21, 1859-1875 (2005).

42. Quinlan, A. R. \& Hall, I. M. BEDTools: a flexible suite of utilities for comparing genomic features. Bioinformatics 26, 841-842 (2010).

43. Kim, D., Paggi, J. M., Park, C., Bennett, C. \& Salzberg, S. L. Graph-based genome alignment and genotyping with HISAT2 and HISAT-genotype. Nat. Biotechnol. 37, 907-915 (2019).

44. Pertea, M. et al. StringTie enables improved reconstruction of a transcriptome from RNA-seq reads. Nat. Biotechnol. 33, 290-295 (2015).

45. Trapnell, C. et al. Transcript assembly and quantification by RNA-Seq reveals unannotated transcripts and isoform switching during cell differentiation. Nat. Biotechnol. 28, 511-515 (2010).

46. Tang, H. et al. Synteny and Collinearity in Plant Genomes. Science 320, 486-488 (2008).

47. Zulkower, V. \& Rosser, S. DNA Features Viewer, a sequence annotations formatting and plotting library for Python. bioRxiv 2020.01.09.900589 (2020) doi:10.1101/2020.01.09.900589. 